\title{
Renewal after the extinction of free operant behavior
}

\author{
Mark E. Bouton • Travis P. Todd • Drina Vurbic • \\ Neil E. Winterbauer
}

Published online: 29 January 2011

(C) Psychonomic Society, Inc. 2011

\begin{abstract}
Four experiments were performed to explore the role of context in operant extinction. In all experiments, leverpressing in rats was first reinforced with food pellets on a variable interval 30 -s schedule, then extinguished, and finally tested in the same and a different physical context. The experiments demonstrated a clear ABA renewal effect, a recovery of extinguished responding when conditioning, extinction, and testing occurred in contexts $\mathrm{A}, \mathrm{B}$, and $\mathrm{A}$, respectively. They also demonstrated $\mathrm{ABC}$ renewal (where conditioning extinction and testing occurred in contexts A, $\mathrm{B}$, and $\mathrm{C}$ ) and, for the first time in operant conditioning, $\mathrm{AAB}$ renewal (where conditioning, extinction, and testing occurred in contexts $\mathrm{A}, \mathrm{A}$, and $\mathrm{B}$ ). The latter two phenomena indicate that tests outside the extinction context are sufficient to cause a recovery of extinguished operant behavior and, thus, that operant extinction, like Pavlovian extinction, is relatively specific to the context in which it is learned. $\mathrm{AAB}$ renewal was not weakened by tripling the amount of extinction training. ABA renewal was stronger than $\mathrm{AAB}$, but not merely because of context A's direct association with the reinforcer.
\end{abstract}

Keywords Extinction · Context · Renewal · Relapse · Instrumental learning $\cdot$ Operant learning

In Pavlovian conditioning, extinction is a decrease in responding that occurs when the conditional stimulus (CS) is repeatedly presented alone, without the unconditional stimulus (US) with which it has been paired. There has been considerable interest in extinction in recent years-in

M. E. Bouton $(\bowtie) \cdot$ T. P. Todd $\cdot$ D. Vurbic $\cdot$ N. E. Winterbauer Department of Psychology, University of Vermont,

Burlington, VT 05405, USA

e-mail: Mark.Bouton@uvm.edu part, because conditioning is thought to play a role in many forms of human psychopathology (e.g., anxiety disorders, drug dependence) and extinction is thus clinically relevant as a way to eliminate unwanted thoughts, emotions, and behaviors (e.g., Bouton, 2002; Craske et al., 2008; Myers \& Davis, 2002). From a practical standpoint, one of the most important things we know about extinction is that it does not merely result from erasure of the original learning. Instead, it at least partly involves a form of new learning that is especially dependent on the context (Bouton, 2004). For example, in the renewal effect, responding to a CS that has been through extinction returns when the CS is tested in a context that is different from the context in which extinction occurred. There are at least three forms of renewal (e.g., Bouton, 2002, 2004). In ABA renewal, conditioning occurs in context $\mathrm{A}$, and extinction occurs in context $\mathrm{B}$. When testing then occurs in context A, responding to the $\mathrm{CS}$ returns. In $\mathrm{ABC}$ renewal, conditioning and extinction occur in contexts $\mathrm{A}$ and $\mathrm{B}$, and recovery is seen in context $\mathrm{C}$. In $\mathrm{AAB}$ renewal, both conditioning and extinction occur in context $\mathrm{A}$, and recovery is seen in context $\mathrm{B} . \mathrm{ABC}$ and $\mathrm{AAB}$ renewal suggest that removal from the extinction context can be sufficient to cause a recovery of responding. The expression of Pavlovian extinction is thus at least partly specific to the context in which it is learned. Renewal and other postextinction response recovery effects may provide mechanisms for understanding relapse after clinical treatment (see Bouton, 2002, 2004).

The present article is concerned with whether extinction after operant learning follows similar principles. In operant learning, the organism associates a behavior with its consequences (typically, reinforcers); in extinction, the behavior declines when the reinforcer is removed. There have been several reports of the ABA renewal effect after operant extinction. For example, Nakajima, Tanaka, Urushihara, and 
Imada (2000) found that rats trained to leverpress for food pellets in one physical context and then extinguished in another physical context showed renewed performance when returned to and tested in the original context (see also Nakajima, Urushihara, \& Masaki, 2002; Welker \& McAuley, 1978). Behavioral pharmacology studies have also documented strong ABA renewal after extinction when rats have been reinforced for leverpressing with intravenous injections of heroin (e.g., Bossert, Liu, Lu, \& Shaham, 2004), cocaine (e.g., Hamlin, Clemens, \& McNally, 2008), a mixture of heroin and cocaine (e.g., Crombag \& Shaham, 2002), or oral delivery of ethanol (e.g., Hamlin, Newby, \& McNally, 2007; Zironi, Burattini, Aicardi, \& Janak, 2006). Such results suggest that drug taking extinguished in one context may relapse when the organism returns to the original context in which the behavior was learned. However, there is little operant research that documents the $\mathrm{ABC}$ and $\mathrm{AAB}$ forms of the renewal effect. Zironi et al. reported preliminary evidence that sucrose-reinforced responding, but not ethanol-reinforced responding, can increase when testing occurs in a novel context after extinction ( $\mathrm{ABC}$ renewal). However, neither Nakajima et al. (2000), Crombag and Shaham, nor Bossert et al. were able to produce an AAB renewal effect. The extent of the parallel between operant extinction and Pavlovian extinction is thus not clear at the present time.

ABA renewal, the effect that has been widely demonstrated in operant experiments, can be caused by any of several mechanisms. First, returning the subjects to a context that has been directly associated with the reinforcer may be sufficient to reinvigorate behavior, because the presence of a Pavlovian CS can enhance operant behavior, as demonstrated by the literature on Pavlovian instrumental transfer (e.g., Balleine, 2005; Colwill \& Rescorla, 1988; Rescorla \& Solomon, 1967). Context-reinforcer associations have also been shown to augment operant responding (e.g., Pearce \& Hall, 1979), including responding that has been eliminated by extinction (i.e., as in reinstatement; Baker, Steinwald, \& Bouton, 1991). Second, it is possible that the leverpress response itself is perceived as different in the two contexts and that extinction in context $\mathrm{B}$ does not directly extinguish the response in context A. Additional findings by Bossert et al. (2004), Crombag and Shaham (2002), and Nakajima et al. (2000) suggest that such a possibility is not necessary for producing ABA renewal: They found no generalization decrement when leverpressing was tested (and extinguished) in context $\mathrm{B}$ after conditioning in context $\mathrm{A}$. A third possibility is that extinction depends on some context-specific form of inhibitory learning that suppresses performance in the extinction context (e.g., Bouton, 2004). But note that ABA renewal does not require this mechanism, because it also involves a return to the original conditioning context. Inhibitory learning is implicated most directly if removal from the extinction context can be shown to be sufficient to cause a recovery of responding. The relative lack of evidence of $\mathrm{ABC}$ or $\mathrm{AAB}$ renewal in operant learning means that there is little unequivocal evidence that operant extinction, like Pavlovian extinction, is relatively specific to the context in which it is learned.

The present experiments were run to help fill this gap in the literature. Each of them used a within-subjects renewal testing method in which rats were tested for operant responding in both the extinction context and another context (in counterbalanced order) after conditioning and extinction (e.g., Bouton \& Ricker, 1994; Rescorla, 2008). In Experiment 1, we found evidence of both $\mathrm{ABA}$ and $\mathrm{AAB}$ renewal. In Experiment 2, we found that tripling the number of extinction sessions did not demonstrably weaken the strength of the AAB renewal effect. In Experiment 3, we found evidence of $\mathrm{ABC}$ renewal. And in Experiment 4, we found that ABA renewal persisted despite extra exposures to context $\mathrm{A}$ (without the operant lever present) that were meant to extinguish context A's direct association with the reinforcer. Together, the results provide a more complete picture of the role of context in operant extinction and indicate that extinction of operant behavior is, in fact, relatively specific to the context in which it is learned.

\section{Experiment 1}

In the first experiment, two groups of rats received training in which a leverpress response was reinforced with a food pellet on a variable interval (VI) 30-s schedule. This training occurred in one context, context A (a chamber from one of two counterbalanced sets of different operant chambers). The groups then received extinction in which the lever was available but presses on it were not reinforced. For group AAB, extinction occurred in context $\mathrm{A}$, the context of conditioning, whereas for group $\mathrm{ABA}$, extinction occurred in context $\mathrm{B}$, the alternate context. Finally, both groups received two test sessions in which responding was tested in both context $\mathrm{A}$ and context $\mathrm{B}$ in a counterbalanced order. If $\mathrm{ABA}$ renewal occurs in this method, the rats in group $\mathrm{ABA}$ were expected to respond more in $\mathrm{A}$ than in $\mathrm{B}$ during testing. If $\mathrm{AAB}$ renewal occurs in this method, the rats in Group $\mathrm{AAB}$ were similarly expected to respond more in context $\mathrm{B}$ than in context $\mathrm{A}$.

In addition to the within-subjects tests emphasized above, the experiment permitted two important comparisons between groups. First, during testing, it allowed a direct comparison of the size of ABA and AAB renewal. Second, during extinction, one group ( $\mathrm{A} A \mathrm{~B})$ received extinction in the context in which leverpressing had been reinforced, and the other group (ABA) received extinction in a different context. This allowed a test of the effect of changing the context on 
operant behavior in the present apparatus and procedure. In Pavlovian extinction, there is often very little loss of performance elicited by the CS when the context is switched after conditioning (e.g., Bouton \& King, 1983; Bouton \& Peck, 1989; Harris, Jones, Bailey, \& Westbrook, 2000), and as was noted above, there is similar evidence of little disruption of operant performance when the context is changed (Bossert et al., 2004; Crombag \& Shaham, 2002; Nakajima et al., 2000). The latter result may be somewhat surprising, in the sense that the context is one of the major cues available to set the occasion for free-operant behavior. In contrast, in the Pavlovian method, the CS directly elicits the response and can potentially do so regardless of the context. In addition, it seems possible that leverpressing might be influenced by subtle differences in the characteristics of the levers in two different apparatuses.

\section{Method}

\section{Subjects}

The subjects were 32 female Wistar rats purchased from Charles River Laboratories (St. Constance, Quebec). They were between 75 and 90 days old at the start of the experiment and were individually housed in suspended wire mesh cages in a room maintained on a 16:8-h light:dark cycle. The rats were food-deprived to $80 \%$ of their initial body weights throughout the experiment. The rats were previously used in a Pavlovian appetitive conditioning experiment in which auditory and visual CSs had been paired with food pellets in a different set of conditioning chambers.

\section{Apparatus}

The apparatus consisted of two unique sets of four conditioning chambers (Med Associates, St. Albans, VT) housed in separate rooms of the laboratory. Each chamber was housed in its own sound attenuation chamber. All boxes measured $30.5 \times 24.1 \times 23.5 \mathrm{~cm}(1 \times \mathrm{w} \times \mathrm{h})$. The sidewalls and ceiling were made of clear acrylic plastic, while the front and rear walls were made of brushed aluminum. The floor was made of stainless steel grids (0.48-cm diameter). A recessed $5.1 \times 5.1 \mathrm{~cm}$ food cup was centered in the front wall approximately $2.5 \mathrm{~cm}$ above the level of the floor. In both sets of boxes, a retractable lever was positioned to the right of the food cup. The lever was $4.8 \mathrm{~cm}$ long and was positioned $6.2 \mathrm{~cm}$ above the grid floor. It protruded $1.9 \mathrm{~cm}$ from the front wall when extended. A $28-\mathrm{V}$ panel light $(2.5 \mathrm{~cm}$ in diameter) was attached to the wall $10.8 \mathrm{~cm}$ above the floor and $6.4 \mathrm{~cm}$ to the left of the food cup. The chambers were illuminated by two $7.5-\mathrm{W}$ incandescent bulbs mounted to the ceiling of the sound attenuation chamber, approximately $34.9 \mathrm{~cm}$ from the grid floor. Ventilation fans provided background noise of $65 \mathrm{~dB}$.

The two sets of four boxes had unique features that allowed them to be used as different contexts (counterbalanced). In one set of boxes, one sidewall had black diagonal stripes, $3.8 \mathrm{~cm}$ wide and $3.8 \mathrm{~cm}$ apart. The ceiling had similarly spaced stripes oriented in the same direction. A distinct odor was continuously presented by placing $5 \mathrm{ml}$ of Pine-Sol (Clorox Co., Oakland, CA) in a dish outside the chamber. The grids of the floor were mounted on the same plane and were spaced $1.6 \mathrm{~cm}$ apart (center to center). The other set of boxes had no distinctive visual cues, and the grids of the floor were staggered such that odd- and evennumbered grids were mounted in two separate planes, one $0.5 \mathrm{~cm}$ above the other. The odor cue was provided by $1.5 \mathrm{ml}$ of Lemon Extract (McCormick, Hunt Valley, MD). The reinforcer was a 45-mg food pellet (Traditional formula, Research Diets, New Brunswick, NJ). The apparatus was controlled by computer equipment located in an adjacent room.

\section{Procedure}

Magazine training On day 1 , each rat was assigned to a box (context A), with the restriction that both sets of boxes were equally represented. All the rats then received a single 30-min session of magazine training in which approximately 60 pellets were delivered randomly on average every $30 \mathrm{~s}$. On day 2, all the rats received magazine training in the other context (context B). The levers were retracted during this phase.

Acquisition On each of the next 5 consecutive days, rats received one session of leverpress training on a VI 30-s reinforcement schedule in context $\mathrm{A}$. The levers were inserted 2 min after the rats had been placed in the chambers. The session ended when the levers were retracted $30 \mathrm{~min}$ later. No additional response shaping was necessary.

Extinction The rats were randomly assigned to two groups ( $n=16$ ), with the restriction that boxes be balanced over the groups. Four daily 30-min sessions of extinction then followed. For group ABA, these sessions occurred in context B. For group AAB, these sessions were conducted in context A. The procedure was exactly the same as the acquisition procedure, except that no reinforcers were delivered.

Renewal test On the final day, the rats received a single 10-min test session in each context. The order of testing was counterbalanced such that half the rats in each group were first tested in context $\mathrm{A}$ and the other half were 
first tested in context B. The two sessions were separated by approximately $60 \mathrm{~min}$. The levers were introduced 2 min after the rats had been placed in the chambers and were retracted $10 \mathrm{~min}$ later. No pellets were delivered in either session.

Data analysis The results were evaluated with analyses of variance (ANOVAs) using a rejection criterion of $p<.05$.

\section{Results}

The left and center portions of Fig. 1 depict response rate (responses per minute) during each session of acquisition and extinction. As is suggested by the figure, rats in both groups acquired the leverpress response quickly and increased their response rates over the five training sessions in context $\mathrm{A}$. This was confirmed by a group $\times$ session ANOVA, which showed a significant session effect, $F(4,120)=114.44$. Neither the group effect nor the group $\times$ session interaction was significant, $F_{\mathrm{S}}<1$. A parallel analysis of extinction indicated that overall, responding declined significantly over sessions, $F(3,90)=185.81$. However, a significant group effect, $F(1,30)=16.56$, and group $\times$ session interaction, $F(3,90)=6.81$, indicated that the ABA group, which was extinguished in a context different from the acquisition context, responded less than the AAB group, which remained in context A during this phase. Thus, with the present apparatus and method, a change of context following acquisition did reduce the level of free-operant leverpressing.

The within-subjects renewal test is shown at the right of Fig. 1. The two test sessions, one of which occurred in the extinction context and one in the renewal context, were analyzed with a group $\times$ context (extinction or renewal) $\times$ order (context A tested first or context B tested first) ANOVA. A significant effect of context confirmed that responding was greater overall in the nonextinction
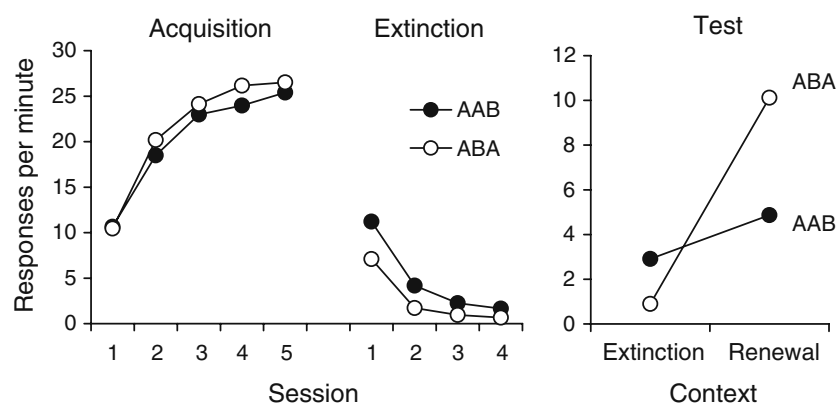

Fig. 1 Results of Experiment 1. Left: Mean responding during each 30-min session of acquisition and extinction. Right: Mean responding during the 10-min test sessions in the extinction context and the nonextinction (renewal) context. $\mathrm{AAB}$, renewal in context $\mathrm{B}$ after extinction in context $\mathrm{A} ; \mathrm{ABA}$, renewal in context $\mathrm{A}$ after extinction in context B (renewal) context, $F(1,28)=64.65$. However, there was also a significant group $\times$ context interaction, $F(1,28)=27.30$. Separate planned analyses of the context effect were conducted for each group. There was a robust renewal effect in the ABA group, $F(1,14)=48.24$, which had a greater than tenfold increase in responding when responding was tested in context A. A parallel comparison for the $\mathrm{AAB}$ group also yielded a significant effect of context, $F(1,14)=22.49$, confirming the occurrence of $\mathrm{AAB}$ renewal; there was a $67.2 \%$ increase in responding in the renewal context. None of the other main effects or interactions in the analyses above were significant, largest $F(1,28)=2.83$. As the figure suggests, group ABA responded more in its nonextinction (renewal) context than did group $\mathrm{AAB}, F(1,30)=11.53$. But in both groups, 16 out of 16 rats $(100 \%)$ responded more in the renewal context than in the extinction context.

\section{Discussion}

The results of this experiment indicate that both $\mathrm{ABA}$ and $A A B$ renewal can occur after operant extinction. The demonstration of $\mathrm{AAB}$ renewal indicates that simple removal from the extinction context is sufficient to create renewal of an operant behavior, as is true of Pavlovian behavior (e.g., Bouton \& Ricker, 1994; Rescorla, 2008; Thomas, Larsen, \& Ayres, 2003). It is noteworthy that $100 \%$ of the rats in the $\mathrm{AAB}$ condition responded more in the renewal context than in the extinction context. The fact that $\mathrm{AAB}$ was numerically weaker than ABA is consistent with several theoretical mechanisms, and it is worth noting that responding in $\mathrm{B}$, a context different from the context in which operant training occurred, was itself weaker than responding in $\mathrm{A}$, as shown during the extinction phase. That result is consistent with the possibility that the context of conditioning sets the occasion for strong operant responding, that context-reinforcer associations acquired during acquisition invigorate the response in context $\mathrm{A}$, or that leverpressing is simply sensitive to subtle differences in the levers in the current different boxes (cf. Bossert et al., 2004; Crombag \& Shaham, 2002; Nakajima et al., 2000). Whatever the cause of the extinction result, it would presumably weaken the strength of any renewal observed in the AAB design.

\section{Experiment 2}

The observation of AAB renewal in Experiment 1 contrasts with the findings of several previous experiments mentioned in the introduction (Bossert et al., 2004; Crombag \& Shaham, 2002; Nakajima et al., 2000). One factor that makes Experiment 1 unique is that renewal was tested in a within-subjects, rather than a between-subjects, manner. 
There are also other factors. For instance, the previous experiments involved more sessions of extinction than we used in Experiment 1. (They also typically involved more acquisition sessions.) Tamai and Nakajima (2000) have shown that $\mathrm{AAB}$ renewal can be eliminated by extended extinction after Pavlovian fear conditioning. Experiment 2 was therefore designed to replicate the operant $\mathrm{AAB}$ renewal effect from Experiment 1 and, furthermore, investigate whether it might be weakened by more extensive extinction training.

Method

\section{Subjects}

The subjects were 32 naive female Wistar rats purchased from the same vendor as those in the previous experiments and maintained under the same conditions.

\section{Apparatus}

The apparatus was the same as that in Experiment 1.

\section{Procedure}

All the phases were carried out in a manner identical to that in Experiment 1, except as noted. Each group received 2 sessions per day throughout the course of the experiment, which were conducted approximately $1 \mathrm{~h}$ apart. Two magazine training sessions (one in each context) occurred on the 1st day, followed by a total of 6 conditioning sessions over the next 3 days. Two groups were then formed so as to match the groups on the total leverpresses before the start of extinction, as well as to counterbalance boxes. For one group (EXT-12, $n=16$ ), extinction began the day following the conclusion of acquisition. This group received 2 extinction sessions on each of the next 6 days, resulting in a total of 12 sessions of extinction. A second group (EXT-4, $n=16$ ) was merely handled on the first 4 days; they then received 2 extinction sessions a day for 2 days, beginning on the 5th day following acquisition. Both groups were then tested the next day ( 7 days after the conclusion of acquisition). Group EXT-12 had received 3 times as many extinction sessions as Group EXT-4.

\section{Results}

The acquisition phase, depicted on the left of Fig. 2, was analyzed with a group $\times$ session ANOVA. A significant effect of session confirmed acquisition of leverpressing, $F(5,150)=122.84$. Not surprisingly, since the groups were matched on their response rates at the end of acquisition, neither the group effect nor the group $\times$ session
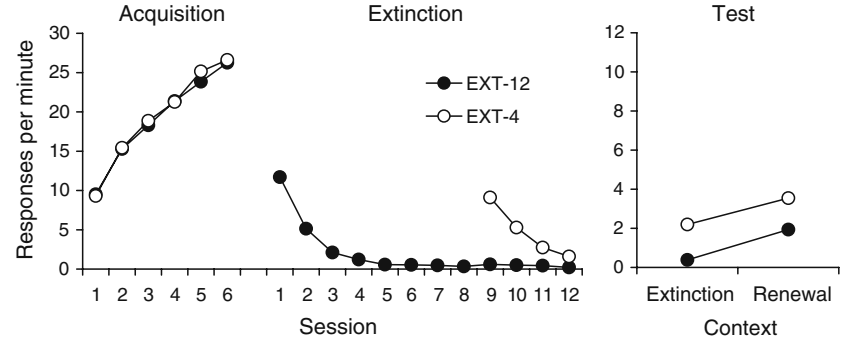

Fig. 2 Results of Experiment 2. Left: Mean responding during each 30-min session of acquisition and extinction. Right: Mean responding during the 10-min test sessions in the extinction context (context A) and the nonextinction (renewal) context (context B). Ext-12, 12 sessions of extinction; Ext-4, 4 sessions of extinction

interaction approached significance, $F \mathrm{~s}<1$. Both groups were subsequently given extinction in context $\mathrm{A}$, which is depicted in the center portion of Fig. 2. A group $\times$ session ANOVA conducted on the first four sessions of each group showed a significant main effect of session, $F(3,90)=224.46$, as well as a group $\times$ session interaction, $F(3,90)=7.87$. The group main effect was not reliable, $F(1,30)<1$. Visual inspection of the figure suggests that group EXT-12 had a higher rate of response at the start of extinction and extinguished more rapidly than group EXT-4, which began the extinction phase 4 days later. Separate ANOVAs were also conducted for the entire extinction phase for each group. Significant effects of session were found in both analyses (group EXT-4, $F(3,45)=129.17$; group EXT-12, $F(11,165)=113.42)$. A follow-up ANOVA isolating the last session of extinction for both groups showed that there was less responding in group EXT-12 than in group EXT-4, $F(1,30)=24.72$.

The data from the tests in contexts $\mathrm{A}$ and $\mathrm{B}$, shown on the right in Fig. 2, were analyzed with a group $\times$ context $\times$ order ANOVA. A significant main effect of context confirmed the presence again of $\mathrm{AAB}$ renewal, $F(1,28)=$ 26.74. Although there was still less overall responding across the two contexts in group EXT-12, $F(1,28)=30.75$, there was no interaction between group and context, $F<1$. Separate context $\times$ order ANOVAs also showed that responding increased in the renewal context for both group EXT-12, $F(1,14)=45.23$, and group EXT-4, $F(1,14)=$ 6.97. None of the other main effects or interactions with order reached significance in any of the analyses, largest $F$ $(1,14)=1.67$. In group Ext-4, 13 out of 16 rats $(81.25 \%)$ responded more in context $\mathrm{B}$ than in context $\mathrm{A}$; in group Ext-12, 14 out of $16(87.50 \%)$ did so.

\section{Discussion}

The results of this experiment replicate the $\mathrm{AAB}$ renewal found in Experiment 1. They also suggest that renewal is not eliminated, or detectably weakened, by tripling the 
amount of extinction training, even though the extra extinction had a demonstrable effect on leverpressing. Although the AAB renewal effect is not numerically large, it is robust in this sense. It is also robust in the sense that, of the total of 48 rats tested in the AAB design in Experiments 1 and 2, $43(89.6 \%)$ demonstrated more responding in context $\mathrm{B}$ (the nonextinction context) than in context $\mathrm{A}$ (the extinction context).

\section{Experiment 3}

In the third experiment, we asked whether $\mathrm{ABC}$ renewal also occurs in the present method. Although the AAB effect observed in Experiments 1 and 2 might make such a result seem likely, the $\mathrm{ABC}$ and $\mathrm{AAB}$ designs differ in that $\mathrm{ABC}$ uniquely provides an opportunity for the rat to learn that extinction occurs after a context switch. That is, in the ABC design, extinction occurs when the animal is moved from the acquisition context (A) to a relatively new context (B); if the relative novelty of the extinction context can become a cue for extinction, extinction in context B might generalize to other relatively novel contexts, reducing any renewal in context $\mathrm{C}$. As was noted earlier, Zironi et al. (2006) found an increase in responding when a sucrosereinforced operant (although not an ethanol-reinforced operant) was tested in a novel context after extinction. However, the contexts were not counterbalanced in that experiment, which makes it possible that other factors (e.g., contextual differences in the ease with which the lever could be operated) could have contributed to the result. It was thus worth asking whether ABC renewal occurs using the present method.

\section{Method}

\section{Subjects}

The subjects were 16 naive female Wistar rats purchased from the same vendor as those in the previous experiments and maintained under the same conditions.

\section{Apparatus}

For all the rats, a new set of four operant chambers (Med Associates, St. Albans, VT) housed in a third room in the laboratory served as context A. These chambers measured $31.8 \times 24.1 \times 29.2 \mathrm{~cm}(1 \times \mathrm{w} \times \mathrm{h})$ and were individually housed in sound attenuation chambers. Ventilation fans provided background noise of $65 \mathrm{~dB}$, and the boxes were lit with two 7.5-W incandescent bulbs mounted to the ceiling of the sound attenuation chamber. The front and back walls were brushed aluminum, while the sidewalls and ceiling were clear acrylic plastic. Recessed $5.1 \times 5.1 \mathrm{~cm}$ food cups were centered in the front wall and positioned near floor level. A 4.8-cm-long stainless steel retractable operant lever protruded $1.9 \mathrm{~cm}$ from the front wall when extended and was positioned $6.2 \mathrm{~cm}$ above the grid floor to the right of the food cup. The floor was composed of stainless steel rods $(0.48 \mathrm{~cm}$ in diameter) spaced $1.6 \mathrm{~cm}$ apart from center to center and mounted parallel to the front wall. The ceiling and left sidewall had black horizontal stripes, $3.8 \mathrm{~cm}$ wide and $3.8 \mathrm{~cm}$ apart. A dish containing $5 \mathrm{ml}$ of a $2 \%$ anise solution (McCormick) was placed outside of each chamber near the front wall.

The lemon-scented and Pine-Sol-scented operant chambers used in the previous experiments served as contexts B and C (counterbalanced).

\section{Procedure}

On day 1, magazine training was conducted, following the usual procedure in all three contexts. Four rats were first trained in context A, 6 were first trained in context B, and 6 were first trained in context C. A minimum of $45 \mathrm{~min}$ elapsed between sessions. Acquisition, extinction, and testing were then carried out, following the procedure used with group ABA in Experiment 1. In the present experiment, all the rats received acquisition training in the anise-scented boxes (context $\mathrm{A}$ ), and then half received extinction in the lemon-scented boxes and half received extinction in the Pine-Sol-scented boxes. Each rat was then given renewal testing, following the usual procedure in both the lemon-scented and Pine-Sol-scented boxes, with test order counterbalanced.

\section{Results}

The results are shown in Fig. 3. As was expected, the rats increased their rate of responding over the five
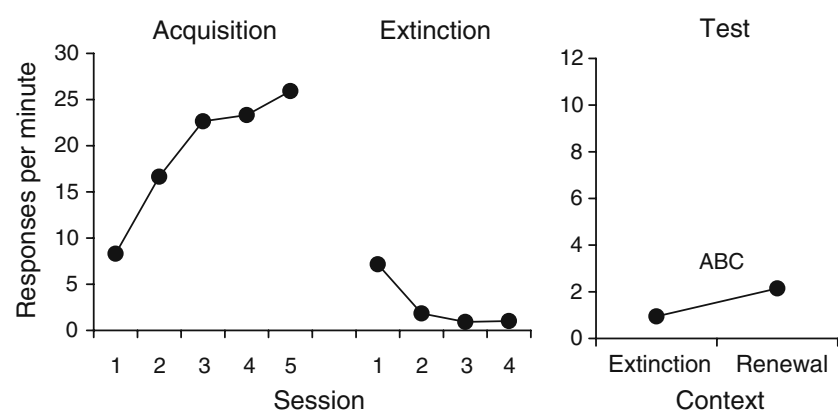

Fig. 3 Results of Experiment 3. Left: Mean responding during each 30-min session of acquisition and extinction. Right: Mean responding during the 10-min test sessions in the extinction context and the nonextinction (renewal) context. $\mathrm{ABC}$, conditioning in context $\mathrm{A}$, extinction in context $\mathrm{B}$, and renewal test in context $\mathrm{C}$ 
acquisition sessions, $F(4,60)=46.25$, and decreased their responding over the four subsequent extinction sessions, $F(3,45)=160.21$. Renewal testing in contexts B and $\mathrm{C}$, which is shown in the right portion of the figure, was analyzed with a context $\times$ order ANOVA. This revealed a significant context effect, $F(1,14)=11.38$, confirming the presence of $A B C$ renewal. Neither the main effect of order nor the context $\times$ order interaction approached significance, $F \mathrm{~s}<1$. Fifteen out of 16 rats $(93.75 \%)$ responded more in context $\mathrm{C}$ than in context $\mathrm{B}$.

\section{Discussion}

These results confirm that $\mathrm{ABC}$ renewal can occur after operant extinction. Like the $\mathrm{AAB}$ result, the $\mathrm{ABC}$ result suggests that a return to the original context of conditioning is not necessary to produce the renewal effect; a test in a context different from the extinction context is sufficient to cause response recovery. Once again, it is worth noting that renewal in a new context occurred despite the fact that operant learning did not generalize perfectly from the original training context to another context in Experiment 1.

\section{Experiment 4}

ABA renewal appears to be stronger than the other forms of renewal investigated here. The explicit comparison between $\mathrm{ABA}$ and $\mathrm{AAB}$ in Experiment 1 supports this, and it is notable that $A B C$ renewal was also not numerically large in Experiment 3. One reason for the relative strength of $\mathrm{ABA}$ renewal is that in the $\mathrm{ABA}$ design, but not the $\mathrm{AAB}$ or $\mathrm{ABC}$ designs, the animal is returned to a context that was associated with the reinforcer during several previous acquisition sessions. Classical conditioning of the background context has long been thought to motivate operant behavior (e.g., Rescorla \& Solomon, 1967), and at least two reports have shown that the conditioning of contextual cues might augment operant behavior (Baker et al., 1991; Pearce \& Hall, 1979).

In the present experiment, we therefore asked whether direct conditioning of context $\mathrm{A}$ during acquisition plays a role in the ABA renewal effect. One group received conditioning in $\mathrm{A}$, extinction in $\mathrm{B}$, and then testing in $\mathrm{A}$ and B. An experimental group received the same treatment but also received four additional sessions of exposure to context A (with levers retracted) for every extinction session it received in context $\mathrm{B}$. The exposures to context A were expected to reduce the impact of a contextreinforcer association through extinction and, thus, reduce any influence of direct conditioning of the context. Extinction exposure to the context has been shown to reduce reinstatement of an extinguished operant response (Baker et al., 1991).

Method

\section{Subjects}

The subjects were 32 female Wistar rats purchased from the same vendor as those in the previous experiments and maintained under the same conditions. They had previously been used in a Pavlovian appetitive conditioning experiment in a different set of conditioning chambers.

\section{Apparatus}

The apparatus was the four anise-scented boxes used in Experiment 3 plus an additional set of four boxes housed in another room. These boxes were similar to the anise-scented boxes, with the same overall construction, dimensions, retractable levers, food cups, and sources of background noise and illumination. However, the floor consisted of alternating stainless steel rods with different diameters $(0.48$ and $1.27 \mathrm{~cm}$ ), spaced $1.6 \mathrm{~cm}$ apart from center to center. The clear acrylic plastic ceiling and left sidewall were covered with rows of dark dots $(1.9 \mathrm{~cm}$ in diameter), each separated by approximately $1.2 \mathrm{~cm}$. And a dish containing $5 \mathrm{ml}$ of $8 \%$ coconut solution (McCormick) was placed outside each chamber near the front wall.

\section{Procedure}

The procedure was the same as that given group $\mathrm{ABA}$ in Experiment 1, except as noted. The groups were matched on their response rates at the end of acquisition, and boxes were fully counterbalanced. During extinction, one group (NoEXP) received the standard daily extinction treatment in context $\mathrm{B}$, while a second group (EXP) received the same treatment with four additional 32-min exposures to context A with the lever retracted. The exposure sessions began at least $1 \mathrm{~h}$ after the extinction session in $\mathrm{B}$; the interval between successive exposure sessions was approximately $40 \mathrm{~min}$. The intervals were spent in the home cage. Rats in group NoEXP received equivalent handling at the same times.

\section{Results}

The data are shown in Fig. 4. Acquisition and extinction of leverpressing were analyzed with separate group $\times$ session ANOVAs. As was expected, significant effects of session were found in both analyses, confirming an increase and subsequent decrease of responding in acquisition and 

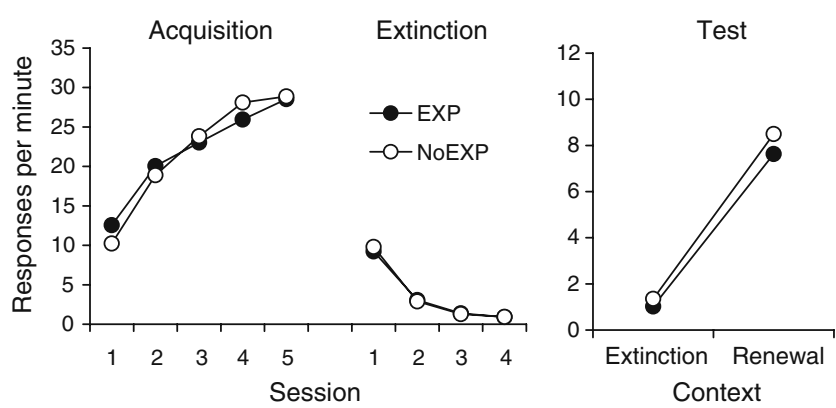

Fig. 4 Results of Experiment 4. Left: Mean responding during each 30-min session of acquisition and extinction. Right: Mean responding during the 10-min test sessions in the extinction context (context B) and the nonextinction (renewal) context (context A). EXP, extra exposures to context A during the extinction phase; NoEXP, no such exposures

extinction. $F(4,120)=86.22$, and $F(3,90)=160.83$. No other main effects or interactions were significant in either analysis, largest $F(4,120)=1.38$.

The renewal test was analyzed with a group $\times$ context $\times$ order ANOVA. As can be seen on the right of Fig. 4, there was a significant effect of context, $F(1,28)=47.48$, confirming an overall ABA renewal effect. However, neither the group effect nor the group $\times$ context interaction approached significance, $F_{\mathrm{S}}<1$. A planned comparison between the two groups on the test in context A also fell well short of the conventional statistical rejection criterion, $F(1$, $28)<1$. There was thus no evidence that the additional exposures to context A weakened renewal in group EXP. Sixteen rats in group NoEXP $(100 \%)$ and 16 rats in group EXP $(100 \%)$ responded more in context A than in context B.

\section{Discussion}

ABA renewal was once again observed in both groups. Although extinction exposure to A should have reduced contextual conditioning in group EXP, it had surprisingly little effect on the renewal effect. Apparently, neither a direct context-reinforcer association nor the relative novelty of the renewing (nonextinction) context plays an important role in producing renewal with the present methods. It is worth noting that one fourth the number of exposures to context $\mathrm{A}$, but with the lever inserted in the chamber, caused a very substantial reduction of responding in context $\mathrm{A}$ in the $\mathrm{AAB}$ groups examined in Experiments 1 and 2. It is also worth noting that analogous exposure to context A likewise does not abolish ABA renewal in either appetitive (e.g., Bouton \& Peck, 1989) or aversive (e.g., Bouton \& Swartzentruber, 1986, 1989) Pavlovian conditioning. Such results may suggest a role for occasion setting by context A, because occasion setting is not abolished by simple extinction (e.g., Rescorla, 1986) or the conditioning of some configural stimulus provided by the response or response lever in combination with context A.

\section{General discussion}

The present experiments used a within-subjects test procedure to document $\mathrm{ABA}, \mathrm{AAB}$, and $\mathrm{ABC}$ renewal after operant extinction. The clear evidence of $\mathrm{AAB}$ and $\mathrm{ABC}$ renewal is especially important, because those effects suggest that operant extinction learning (like Pavlovian extinction learning) is relatively specific to the context in which it is learned.

The finding that responding is renewed when the response is tested outside the extinction context is consistent with the idea that the context of extinction somehow inhibits the behavior. One account of renewal is that the switch out of the extinction context disinhibits the extinguished response (e.g., Brimer, 1970). It is worth noting that the term disinhibition tends to describe a phenomenon, rather than an underlying behavioral process, and that the phenomenon itself is not well understood. Because the procedures that induce disinhibition can be conceptualized as procedures that also change the context, disinhibition may be an example of the renewal effect.

How are we to characterize the inhibitory process by which the context inhibits extinguished behavior? One possibility is that the animal learns an inhibitory association between the situation and the operant response. Rescorla (1993, 1997, 2001) has reported a number of results that are consistent with such a mechanism. For example, Rescorla (1993) reinforced two behaviors and then extinguished each in combination with its own stimulus (either a light or a noise). Subsequent tests revealed that each response was suppressed the most when it was tested with the stimulus in which it had been extinguished (see also Rescorla, 1997). Such results are clearly compatible with the present demonstrations of renewed responding outside the context of extinction. Although they are consistent with the possibility that the rat might learn an inhibitory context-response association, they are also consistent with the idea that the rat learns that the stimulus set the occasion for a response-no-reinforcer relationship (see Bouton, 2004; Rescorla, 1993 [p. 335], 1997 [p. 249]). It is worth noting that the inhibitory S-R mechanism has recently come into some question. Although the mechanism has been thought to explain why procedures that lead to high levels of responding during extinction generate a stronger and more durable extinction result (Rescorla, 1997, 2001), the strength of renewal and spontaneous recovery in Pavlovian learning have been reported to be positively (rather than negatively) correlated with the level of responding observed in extinction (Bouton, Garcia-Gutiérrez, Zilski, \& Moody, 
2006; Moody, Sunsay, \& Bouton, 2006). And Rescorla (2006, Experiment 5) has shown that high levels of responding do not guarantee a durable form of extinction.

The corresponding literature on Pavlovian renewal has instead emphasized the occasion-setting mechanism (e.g., Bouton, 2004). There, experimenters have considered the possibility that the extinction context is either a simple conditioned inhibitor (with a direct inhibitory association with the US) or a negative occasion setter (e.g., a signal for a CS-no-US relationship). A number of experiments have failed to uncover simple inhibition to the extinction context (Bouton \& King, 1983; Bouton \& Swartzentruber, 1986, 1989). That, coupled with sparse evidence that CSs and contexts summate (e.g., Bouton, 1984), has favored the hypothesis that the context of extinction is a negative occasion setter.

The finding that removal from the extinction context may be sufficient to cause renewal might help explain other findings. For instance, Winterbauer and Bouton (2010) recently examined a variation on the traditional instrumental extinction procedure in which a second instrumental response is reinforced while a previously trained response is extinguished. When the second behavior is then extinguished, the first behavior may recover or "resurge" (e.g., Leitenberg, Rawson, \& Bath, 1970). Winterbauer and Bouton suggested that this phenomenon might be a form of $\mathrm{ABC}$ renewal: The first response is trained in the absence of the second one (context A) and then extinguished while the second response is reinforced (context $\mathrm{B}$ ). When the second response is then itself extinguished, it creates a third set of conditions (context C) that might allow recovery of the first response. Documentation of $\mathrm{ABC}$ renewal in the present article suggests that such an analysis is plausible.

In addition to some form of inhibitory control by the extinction context, the fact that renewal was especially strong in the ABA design (Experiment 1) suggests that the context of conditioning (context A) might also modulate extinguished responding. One possibility is that direct conditioning of context A excited the response. Direct conditioning of the context could have also contributed to the present $\mathrm{AAB}$ and $\mathrm{ABC}$ renewal effects, because the rats had received a relatively small number of food pellets in the renewal contexts as part of their initial magazine training. Although there is evidence that contextual conditioning can indeed invigorate operant responding (Baker et al., 1991; Pearce \& Hall, 1979), the fact that extinction exposures to context A did so little to weaken ABA renewal in Experiment 4 suggests that simple context conditioning might have played little role in the present experiments. Of course, Experiment 4 used only one method to test the effect of direct context-reinforcer associations and only one set of acquisition and extinction parameters. It is possible that a stronger role for context-reinforcer associations would have emerged, for example, if a richer schedule of reinforcement had been used in the acquisition phase. However, a second possibility is that context A plays the role of a positive occasion setter - that is, a stimulus that selects or enables the response-reinforcer relationship (e.g., Rescorla, 1991). As was noted earlier, occasion setters are not strongly affected by simple extinction (Experiment 4). A third possibility is that a configural cue that combines elements of both the response and context A might control the response. One way to think of such a configural cue is to suppose that leverpressing is partly a result of Pavlovian conditioning of the lever stimulus. On this view, the rat might associate the lever with food during instrumental training; it might, therefore, evoke Pavlovian approach and manipulation (e.g., consummatory) behaviors (e.g., Bindra, 1972; Bolles, 1972; Timberlake, 2001). On a configural account, the lever stimulus in context A might be strongly excitatory, whereas the lever stimulus in context $\mathrm{B}$ might become inhibitory (e.g., Pearce, 1994). While such a view is consistent with ABA renewal (and the decrease in responding observed after switching from context $\mathrm{A}$ to $\mathrm{B}$ ), it does not predict $\mathrm{AAB}$ or $\mathrm{ABC}$ renewal, because there is no expectation that excitation acquired by the configural stimulus during conditioning would generalize more to a new context than would the inhibition acquired by the configural stimulus present during extinction. It is worth noting that the possible role of Pavlovian learning with the lever stimulus is also compatible with the context-as-excitor and context-as-occasion-setter mechanisms. A more complete understanding of the influence of context $\mathrm{A}$ in operant renewal will require additional research.

The possibility that operant extinction is relatively specific to the context in which it is learned has implications for the clinical treatment of voluntary behavior, such as various forms of substance abuse. The context specificity of extinction would allow an extinguished behavior to lapse when it could occur in any nonextinction context. Furthermore, the possibility that simple extinction exposure to the context of original training might not eliminate renewal (Experiment 4) suggests that simple cue exposure treatments might not always be effective at eliminating the operant behavior (see also Conklin \& Tiffany, 2002). Finally, it is worth noting that in the world outside the laboratory, when a drug abuser undergoes a renewal effect, the "test" trials are generally reinforced; the smoker smoking a cigarette and the drinker taking a drink are reexposed to the response-reinforcer contingency. In contrast, the renewal effects observed here were always assessed in tests conducted in extinction. When coupled with reconditioning, they could easily begin the spiral into relapse. Renewal, like other response recovery effects known to occur after operant extinction (such as reinstatement, spontaneous recovery, and resurgence), might con- 
tribute to the fact that voluntary, operant behaviors are notoriously resistant to change.

\section{References}

Baker, A. G., Steinwald, H., \& Bouton, M. E. (1991). Contextual conditioning and reinstatement of extinguished instrumental responding. The Quarterly Journal of Experimental Psychology, $43 B, 199-218$

Balleine, B. W. (2005). Neural bases of food-seeking: Affect, arousal and reward in corticostriatolimbic circuits. Physiology \& Behavior, 86, $717-730$

Bindra, D. (1972). A unified account of classical conditioning and operant training. In A. H. Black \& W. F. Prokasy (Eds.), Classical conditioning II: Current research and theory (pp. 453-481). New York: Appleton-Century-Crofts.

Bolles, R. C. (1972). Reinforcement, expectancy, and learning. Psychological Review, 79, 394-409.

Bossert, J. M., Liu, S. Y., Lu, L., \& Shaham, Y. (2004). A role of ventral tegmental area glutamate in contextual cue-induced relapse to heroin seeking. The Journal of Neuroscience, 24, 10726-10730.

Bouton, M. E. (1984). Differential control by context in the inflation and reinstatement paradigms. Journal of Experimental Psychology: Animal Behavior Processes, 10, 56-74.

Bouton, M. E. (2002). Context, ambiguity, and unlearning: Sources of relapse after behavioral extinction. Biological Psychiatry, 52, 976-986.

Bouton, M. E. (2004). Context and behavioral processes in extinction. Learning \& Memory, 11, 485-494.

Bouton, M. E., García-Gutiérrez, A., Zilski, J., \& Moody, E. W. (2006). Extinction in multiple contexts does not necessarily make extinction less vulnerable to relapse. Behaviour Research and Therapy, 44, 983-994.

Bouton, M. E., \& King, D. A. (1983). Contextual control of the extinction of conditioned fear: Tests for the associative value of the context. Journal of Experimental Psychology: Animal Behavior Processes, 9, 248-265.

Bouton, M. E., \& Peck, C. A. (1989). Context effects on conditioning, extinction, and reinstatement in an appetitive conditioning preparation. Animal Learning \& Behavior, 17, 188-198.

Bouton, M. E., \& Ricker, S. T. (1994). Renewal of extinguished responding in a second context. Animal Learning \& Behavior, 22, 317-324.

Bouton, M. E., \& Swartzentruber, D. (1986). Analysis of the associative and occasion-setting properties of contexts participating in a Pavlovian discrimination. Journal of Experimental Psychology: Animal Behavior Processes, 12, 333-350.

Bouton, M. E., \& Swartzentruber, D. (1989). Slow reacquisition following extinction: Context, encoding, and retrieval mechanisms. Journal of Experimental Psychology: Animal Behavior Processes, 15, 43-53.

Brimer, C. J. (1970). Disinhibition of an operant response. Learning and Motivation, 1, 346-371.

Colwill, R. M., \& Rescorla, R. A. (1988). Associations between the discriminative stimulus and the reinforcer in instrumental learning. Journal of Experimental Psychology: Animal Behavior Processes, 14, 155-164.

Conklin, C. A., \& Tiffany, S. T. (2002). Applying extinction research and theory to cue-exposure addiction treatments. Addiction, 97, 155-167.

Craske, M. G., Kircanski, K., Zelikowsky, M., Mystkowski, J., Chowdhury, N., \& Baker, A. (2008). Optimizing inhibitory learning during exposure therapy. Behaviour Research and Therapy, 46, 5-27.
Crombag, H. S., \& Shaham, Y. (2002). Renewal of drug seeking by contextual cues after prolonged extinction in rats. Behavioral Neuroscience, 116, 169-173.

Hamlin, A. S., Clemens, K. J., \& McNally, G. P. (2008). Renewal of extinguished cocaine-seeking. Neuroscience, 151, 659-670.

Hamlin, A. S., Newby, J., \& McNally, G. P. (2007). The neural correlates and role of D1 dopamine receptors in renewal of extinguished alcohol-seeking. Neuroscience, 146, 525-536.

Harris, J. A., Jones, M. L., Bailey, G. K., \& Westbrook, R. F. (2000). Contextual control over conditioned responding in an extinction paradigm. Journal of Experimental Psychology: Animal Behavior Processes, 26, 174-185.

Leitenberg, H., Rawson, R. A., \& Bath, K. (1970). Reinforcement of competing behavior during extinction. Science, 169, 301-303.

Moody, E. W., Sunsay, C., \& Bouton, M. E. (2006). Priming and trial spacing in extinction: Effects on extinction performance, spontaneous recovery, and reinstatement in appetitive conditioning. The Quarterly Journal of Experimental Psychology, 59, 809-829.

Myers, K. M., \& Davis, M. (2002). Behavioral and neural analysis of extinction. Neuron, 36, 567-584.

Nakajima, S., Tanaka, S., Urushihara, K., \& Imada, H. (2000). Renewal of extinguished lever-press responses upon return to the training context. Learning and Motivation, 31, 416-431.

Nakajima, S., Urushihara, K., \& Masaki, T. (2002). Renewal of operant performance formerly eliminated by omission or noncontingency training upon return to the acquisition context. Learning and Motivation, 33, 510-525.

Pearce, J. M. (1994). Similarity and discrimination: A selective review and a connectionist model. Psychological Review, 101, 587-607.

Pearce, J. M., \& Hall, G. (1979). The influence of context-reinforcer associations on instrumental performance. Animal Learning \& Behavior, 7, 504-508.

Rescorla, R. A. (1986). Extinction of facilitation. Journal of Experimental Psychology: Animal Behavior Processes, 12, 16-24.

Rescorla, R. A. (1991). Associative relations in instrumental learning: The Eighteenth Bartlett Memorial Lecture. The Quarterly Journal of Experimental Psychology, 43B, 1-23.

Rescorla, R. A. (1993). Inhibitory associations between S and R in extinction. Animal Learning \& Behavior, 21, 327-336.

Rescorla, R. A. (1997). Response inhibition in extinction. The Quarterly Journal of Experimental Psychology, 50B, 238-252.

Rescorla, R. A. (2001). Experimental extinction. In R. R. Mowrer \& S. B. Klein (Eds.), Handbook of contemporary learning theories (pp. 119-154). Mahwah, NJ: Erlbaum.

Rescorla, R. A. (2006). Deepened extinction from compound stimulus presentation. Journal of Experimental Psychology: Animal Behavior Processes, 32, 135-144.

Rescorla, R. A. (2008). Within-subject renewal in sign tracking. The Quarterly Journal of Experimental Psychology, 61, 1793-1802.

Rescorla, R. A., \& Solomon, R. L. (1967). Two-process learning theory: Relationships between Pavlovian conditioning and instrumental learning. Psychological Review, 74, 151-182.

Tamai, N., \& Nakajima, S. (2000). Renewal of formerly conditioned fear in rats after extensive extinction training. International Journal of Comparative Psychology, 13, 137-147.

Thomas, B. L., Larsen, N., \& Ayres, J. J. B. (2003). Role of context similarity in $\mathrm{ABA}, \mathrm{ABC}$, and $\mathrm{AAB}$ renewal paradigms: Implications for theories of renewal and for treating human phobias. Learning and Motivation, 34, 410-436.

Timberlake, W. (2001). Motivational modes in behavior systems. In R. R. Mowrer \& S. B. Klein (Eds.), Handbook of contemporary learning theories (pp. 155-210). Mahwah, NJ: Erlbaum.

Welker, R. L., \& McAuley, K. (1978). Reductions in resistance to extinction and spontaneous recovery as a function of changes in transportational and contextual stimuli. Animal Learning \& Behavior, 6, 451-457. 
Winterbauer, N. E., \& Bouton, M. E. (2010). Mechanisms of resurgence of an extinguished instrumental behavior. Journal of Experimental Psychology: Animal Behavior Processes, 36, 343-353.

Zironi, I., Burattini, C., Aicardi, G., \& Janak, P. H. (2006). Context is a trigger for relapse to alcohol. Behavioural Brain Research, 167, $150-155$.
This research was supported by Grant R01 MH064847 from the National Institute of Mental Health. TPT was partially supported by a graduate scholarship from the National Science and Engineering Research Council of Canada. Send correspondence to Mark E. Bouton, Department of Psychology, University of Vermont, 2 Colchester Ave., Burlington, VT 05405. 